\title{
EVOLUCIÓN HISTÓRICA DE LA EVALUACIÓN EN LA EDUCACIÓN SUPERIOR ECUATORIANA: PASOS HACIA LA EXCELENCIA.
}

\author{
THE HISTORICAL EVOLUTION OF THE EVALUATION IN HIGHER \\ EDUCATION IN ECUADOR: STEPS TOWARDS EXCELLENCE.
}

\author{
Sandra Canalías Lamas, MS.c \\ sandra_canalias@hotmail.com \\ Docente Carrera de Derecho UPSE \\ Margarita Lamas González, Ph.D \\ Docente Investigador ORDECO UPSE
}

\section{RESUMEN}

El desarrollo que ha tenido la Educación Superior en el mundo y las exigencias actuales de las sociedades, requiere de acercarnos a modelos de evaluación que transiten desde lo institucional hasta lo profesional de los docentes universitarios. En el presente artículo se realizan valoraciones de la evolución histórica de la evaluación de las casas de altos estudios en la República del Ecuador, hasta la actualidad. Se utilizaron los métodos y técnicas desde la perspectiva de la investigación cualitativa, identificando la necesidad de realizar evaluaciones que las lleven hacia la excelencia.

Palabras clave: Evaluación, educación superior.

\section{ABSTRACT}

The developments in higher education around the world and the current demands in our societies require us to consider evaluation models that travel from the institutional to the professional ranks of university professors. In this article, there will be an analysis of the historical evolution of the institutions of higher learning in the Republic of Ecuador. Methods and techniques were used from the perspective of a qualitative investigation, identifying the need to perform evaluations that will drive towards excellence.

Keywords: higher education, evaluations

Recibido: octubre de 2014 Aprobado: noviembre de 2014

\section{Introducción}

Acercarnos a los procesos evaluativos y de acreditación de la Educación Superior en la República de Ecuador, es adentrarnos en la evolución histórica que han tenido las universidades, posterior a la etapa colonial, cuando en el año 1826, por decreto del Libertador Simón Bolívar, se fundó la Universidad de Quito. Fue en su salón de actos, el 13 de mayo de 1830, donde se firmó el Acta de Separación de la Gran Colombia y se constituyó la República del Ecuador.

En 1835, cinco años después, de haberse iniciado la etapa Republicana, se establece la Dirección General de Estudios y las subdirecciones de Inspectorías y de Instrucción. La instrucción pública se inició en establecimientos fiscales y en los de órdenes religiosas, denominadas escuelas primarias, secundarias y universidades.

Posteriormente, “...en 1862 llegaron los sacerdotes a esta República por segunda vez. La Convención Nacional admitió a los jesuitas para que ayudaran al progreso de la enseñanza".(1) Con la llegada de estos se restableció su influencia religiosa y su enseñanza al hacerse cargo de los colegios de segunda enseñanza y de los seminarios de Quito, Guayaquil, Riobamba, Cuenca, entre otros. "En el año 1870 los jesuitas iniciaron los cursos de la Escuela Politécnica; dan evidencia de esta gestión, las obras y los diversos trabajos que publicaron sus profesores y los programas por los que los alumnos se exa- 
minaban...”.(2)

La Dirección General de Estudios pasó a denominarse Consejo General de Instrucción Pública, a partir de 1875, en que se le asignó la responsabilidad de la administración de las universidades, los colegios, los liceos y las escuelas, conforme a la doctrina de la religión católica.

La Universidad Central del Ecuador se cerró en el año 1869 y en su lugar se estableció, bajo la dirección de los jesuitas, la primera Escuela Politécnica del Ecuador, la que contó con instalaciones modernas, como un observatorio astronómico y un jardín botánico. En 1883, se restableció la Universidad Central del Ecuador con nuevos recursos, entre ellos un microscopio. Sin duda, estos avances tecnológicos evidenciaron un interés por lograr medios de apoyo para la enseñanza universitaria; no obstante, al menos hasta finales del siglo XIX, no se proyectaron precisiones relativas a la valoración y al mejoramiento del quehacer de los profesores universitarios, tanto, desde aquellas instituciones o desde el Consejo General de Instrucción Pública.

En 1901, le fue retirada a los jesuitas la enseñanza oficial. Los estudios realizados revelaron que, además de la propagación del evangelio, en los dos períodos de sus misiones, se hicieron grandes esfuerzos por la instrucción. En ese entonces, la enseñanza universitaria se dedicó, fundamentalmente, a la medicina, la teología, el derecho civil, las letras, las artes y la arquitectura. Numerosas obras existentes en todo el Ecuador, dan fe de la acción jesuita en las universidades. "Sin género de duda, en Quito fueron los jesuitas los que más contribuyeron al realce cultural y moral, mediante el Colegio de San Luis y la Universidad de San Gregorio. Ésta fue el semillero del saber y la cultura para eclesiásticos, religiosos y civiles. Los jesuitas fueron los que más influyeron en la cultura de Quito, tanto por la competencia de su profesorado como por los libros que dejaron escritos".(3)

No obstante, los avances en el desarrollo de las actividades universitarias en esta etapa, durante toda la gestión de los jesuitas en la conducción de la educación universitaria, la calidad de la instrucción no constituyó una preocupación; de lo que se infiere que la labor pedagógica de los profesores y las acciones para su mejoramiento, no fueran aspectos valorados en los niveles que se requerían, para asegurar una instrucción y educación de calidad, por lo que, la evaluación institucional no poseía un antecedente claro, desde los comienzos de la Educación Superior. En el presente artículo, centramos la atención en los primeros intentos de realizar normas que tuvieran la intención de evaluar las universidades, desde su funcionamiento como institución de altos estudios, hasta la evaluación del desempeño de sus docentes.

\section{DESARROLLO.}

La sistematización realizada, posterior a la etapa colonial, en Ecuador, nos acerca a conocer que la educación era una cuestión prioritaria del gobierno liberal. Así, en el año 1906, se declara la oficialidad de la educación universitaria laica. En ese marco, se emprende la reforma educativa más exitosa de la historia nacional, a través de la creación de las Escuelas Normales, como parte de la Ley Orgánica de Instrucción Pública (4). Mediante esta Ley se determinó que la instrucción se diera en todos los establecimientos nacionales sostenidos por el Estado, comprendiendo la enseñanza primaria, secundaria y superior, y organizada en escuelas, colegios y universidades, respectivamente. La enseñanza superior comprendió, entonces, las facultades de Jurisprudencia; Medicina, Cirugía y Farmacia; y Ciencias Matemáticas, Físicas y Naturales. Por esta ley, se institucionalizaron, también, los establecimientos de enseñanza libre, sostenidos por corporaciones o por personas y/o entidades particulares, incluyendo universidades.

En 1906, se expide el Reglamento General de las Escuelas Normales. Según refiere Ayala E (5), para incentivar la matrícula se crearon becas y la formación docente, mediante cursos intensivos. En ese entonces, "...la enseñanza de las asignaturas pedagógicas era todavía, incipiente; en la Normal de Varones sólo había una hora semanal de Pedagogía en la sección superior y en la Normal de Señoritas se enseñaba Psicología, sólo una hora por semana".(6)

Según, refiere Pareja F (7) en su monografía Educación Superior del Ecuador, la Ley de Educación Superior del año 1938 otorgó a las universidades ecuatorianas autonomía para su funcionamiento técnico y administrativo. A pesar, de los importantes avances cuantitativos y cualitativos que se establecieron durante las décadas del 30 al 50 del pasado siglo; a fines de los años 50 , persistían hechos que evidenciaban las insuficiencias del sistema universitario ecuatoriano, entre ellas: “... gestión educativa divorciada de las necesidades particulares de la comunidad; programas de estudios con contenidos disfuncionales; inestabilidad del docente; limitada capacidad física instalada para facilitar el acceso a este nivel educativo; altos índices de repetición y deserción; bajo rendimiento interno; y, escasa productividad externa". (8) 
Las limitaciones referidas, revelan que, a finales de los años 50 del pasado siglo, predominaban insuficiencias en el quehacer pedagógico de los profesores universitarios y se daba poco énfasis en la necesidad de potenciar el mejoramiento de su desempeño, tanto, por parte, de las autoridades institucionales, como de las oficiales. No obstante, fundamentalmente, en la segunda mitad del siglo y derivado de la influencia de la modernidad, surgieron en todo el territorio del Ecuador una serie de universidades, entre las que se encuentra la Universidad Católica de Cuenca, fundada el 7 de octubre de 1970.

En la década de los 60, el Ministerio de Educación inició un proceso de modernización institucional, con la creación del Departamento de Planeamiento Integral. Entre los años sesenta y ochenta, este ministerio consolidó su gestión, sobre todo, para solucionar los problemas que determinaban las insuficiencias educacionales, incluyendo las que se manifestaban en las universidades. Sin embargo, estas transformaciones no se hacían sobre bases sólidas de medición del funcionamiento de las universidades o de valoración el desempeño de sus profesores.

Un importante hito histórico de la educación ecuatoriana, lo constituyó la puesta en práctica de un proyecto orientado al mejoramiento de la calidad de la educación. Este proyecto, que abarcó, prácticamente, toda la década de los años 90, partió de ejes problemáticos fundamentales, entre los que se encontraban algunos de los que revelaron las insuficiencias existentes en la formación y la superación de los docentes y la falta de atención al desarrollo de alternativas que favorecieran el mejoramiento de su desempeño profesional pedagógico, a finales del pasado siglo. Entre las referidas por Abendaño A, (9) las más vinculadas a este aspecto fueron:

- Escasez de control sobre el ejercicio de los docentes, debido a la inexistencia de un sistema de evaluación de la calidad y de asesoría pedagógica.

- Falta de indicadores de calidad en los establecimientos y niveles, lo cual impide evaluar y hacer el seguimiento de los procesos educativos.

- Carencia de prácticas educativas y de un sistema de dinamización del pensamiento pedagógico, debido a la falta de eventos y publicaciones que estimulen la investigación y la renovación educativas.

- Debilidades en la formación inicial y desarrollo de los recursos humanos que laboran en todo el sector educativo, incluyendo las universidades.

- Deterioro de la autoimagen del docente, originado por la ausencia de mecanismos que lo estimulen a competir y mejorar, profesionalmente.

- Inexistencia de un sistema equitativo de ingreso, promoción y estimulación a los profesores, así como carencia de mecanismos de control de las prácticas docentes.

Muchas de las problemáticas asociadas con la calidad de la educación superior, en la dimensión del desempeño de sus docentes, aún subsisten en las universidades ecuatorianas.

La transformación de las escuelas normales en institutos pedagógicos, en 1991, fue uno de los resultados del referido proyecto. A estos Centros se les asignó funciones complementarias, como: la investigación y experimentaciones pedagógicas, la producción de recursos didácticos y la extensión educativa en la comunidad.

Estas reformas, en la formación de docentes, tuvieron un impacto positivo, sobre todo en el crecimiento del número de profesores. Ello, coincidió con reformas orientadas al fortalecimiento del sector público en la Educación Superior del Ecuador, lo que implicó el acceso de un significativo número de estudiantes de sectores populares, hasta, entonces, excluidos. Ante esta situación, entidades particulares emprendieron la fundación de una nueva oleada de universidades privadas con el interés de mantener la excelencia y exclusividad de los servicios universitarios para las clases altas de la sociedad ecuatoriana.

La formación de profesores ha tenido en el país un largo proceso. Cada una de las reformas cumplió con sus objetivos específicos en momentos históricos determinados; no obstante, en general, se dieron pocos pasos de avances respecto a la profesionalización pedagógica de los docentes. En el caso de los profesores universitarios, al menos hasta la década de los años 90 del pasado siglo, no existió una política estatal que direccionara el postgrado como forma explícita de superación, orientada al mejoramiento de su desempeño profesional pedagógico. En estos años, no existía un consenso, ni acuerdos nacionales sobre qué desempeños se deberían esperar de los profesionales de la educación, ni cómo deberían funcionar, óptimamente, las instituciones educativas. Una consecuencia de la falta de criterios de calidad educativa, fue la inexistencia de un perfil común para la formación de los docentes, en las universidades e institutos pedagógicos.

En esta etapa, la educación de postgrado respondía a iniciativas de algunas autoridades y universidades. Se caracterizó por el predominio de inte- 
reses individuales, la falta de sistematicidad y una insuficiente coherencia de las acciones.

Hasta la década de los años 90, se observó que los docentes de los Centros de la Educación Superior, poseían una insuficiente formación básica o especializada, en el área pedagógica, al no ser graduados en pedagogía o ciencias de la educación y al no haber recibido superación o capacitación continua, en esta área de las ciencias.

Dado el auge y la expansión internacional del postgrado, durante la década de los 90, creció el interés por esta importante forma de superación y por el mejoramiento profesional de los egresados universitarios en servicio, incluyendo los docentes universitarios. Es así que, el postgrado se convirtió en un aspecto fundamental en el funcionamiento de las universidades, como vía de superación profesional y de interrelación con entidades públicas y privadas.

La educación de postgrado estuvo centrada en ejecutar convenios regionales, nacionales e internacionales de cooperación para la organización e intercambio de programas de especialización, diplomados, maestrías y doctorados. No obstante, los docentes de las universidades, generalmente, sin formación pedagógica, se superaban en sus respectivas áreas y no recibían preparación postgraduada orientada al mejoramiento de su desempeño profesional pedagógico.

En el año 2000, se expidió una nueva Ley de Educación Superior (10); en ella se determinó que formaban parte del Sistema Nacional de Educación Superior, las universidades, las escuelas politécnicas y los institutos superiores técnicos y tecnológicos incorporados al sistema. "Estas instituciones tienen como misión la búsqueda de la verdad, el desarrollo de la cultura universal y ancestral ecuatoriana, la ciencia y la tecnología, mediante la docencia, la investigación y la vinculación con la colectividad, con el objeto de responder con pertinencia al desarrollo del país".(11)

En correspondencia con este encargo, se crearon y fortalecieron las estructuras administrativas del Consejo Nacional de Universidades y Escuelas Politécnicas (CONESUP), las que asumieron importantes funciones y responsabilidades. Cabe indicar que ninguna se refirió, explícitamente, a la superación pedagógica de los profesores universitarios; ésta quedó como atribución de las instituciones de educación superior, sobre las cuales se proyectó el interés de promover y apoyar la investigación científica, así como la gestión para su desarrollo interno y para la transferencia de resultados a la sociedad.
Bajo la influencia del neoliberalismo, surgieron en el Ecuador, universidades y carreras que mercantilizaron la educación superior, sin que en ellas se tuvieran en cuenta criterios de calidad que aseguraran procesos de formación profesional que respondieran a las necesidades sociales y al desarrollo del país. El crecimiento de universidades determinó la disminución del control estatal de la calidad en la gestión universitaria, incluyendo el desempeño profesional de los docentes.

Hasta el año 2007, no se orientó una verdadera política oficial sobre el control de la calidad de los procesos universitarios, ni de la superación de los profesionales que se desempeñaban como docentes en las instituciones de educación superior en el Ecuador, no obstante, a que ello estuvo implícito en lo reglamentado en la Ley de Universidades y Escuelas Politécnicas y en el quehacer del CONESUP.

Según, un Informe del Consejo Nacional de Evaluación y Acreditación de la Educación Superior (CONEA), referido por Macías W., "... la planta docente del conjunto de universidades ecuatorianas en el 2008 sumaban un total de 33.007 profesores, esto es para las 68 universidades en funcionamiento (...). En ese entonces, tan solo existían 482 doctores en el ámbito universitario y politécnico, lo que representa el 3,3\%".(12)

Aún, cuando en la Ley de Educación Superior del 2000 se señaló el establecimiento de un sistema de evaluación y acreditación de la Educación Superior, no fue hasta después que se estructuró y estableció el CONEA. Éste empezó a laborar en forma autónoma e independiente, pero en coordinación con el CONESUP, hasta la eliminación de este organismo y la creación del Consejo de Educación Superior (CES).

"Ecuador llegó con retraso al proceso de aseguramiento de la calidad de la educación superior. Mientras, que en los años 80 y 90, varios países poseían organismos especializados encargados en asegurar la calidad; en Ecuador se debatía la calidad y se reconocía que había un deterioro cualitativo de la educación superior, como resultado de un conjunto de factores, entre ellos: la insuficiencia de recursos para el desarrollo de los programas académicos; el rápido crecimiento de la matrícula universitaria y el número de universidades; la política de admisión, ligada al deterioro de la Educación Media; el bajo componente de académicos a tiempo completo; la captación de egresados, sin formación de cuarto nivel, para ejercer la docencia; la insuficiente infraestructura tecnológica; la débil vinculación de las universidades con el desarrollo científico-tecnológico y la 
insuficiente asignación de recursos para el desarrollo de investigaciones".(13)

Con el gobierno del presidente Rafael Correa, en abril de 2008, se inició una etapa de cambios sociales e institucionales. Por primera vez en la Constitución de la República se explicita el objetivo supremo de "...avanzar hacia una sociedad democrática, soberana, justa, incluyente, intercultural, plurinacional y segura, con personas libres, autónomas, solidarias, creativas, equilibradas, honestas, trabajadoras y responsables, que antepongan el bien común al bien individual, que vivan en armonía con los demás y con la naturaleza". (14)

Al amparo de la nueva Constitución y frente a la situación existente, en el año 2010 se constituyó la actual Ley Orgánica de Educación Superior (LOES), que en su artículo 4 plantea el derecho a este sistema educativo, como "....ejercicio efectivo de igualdad de oportunidades, en función de los méritos respectivos, a fin de acceder a una formación académica y profesional con producción de conocimiento pertinente y de excelencia".(15) Entre los fines planteados a esta educación, se llama a aportar al desarrollo del pensamiento universal, al despliegue de la producción científica y a la promoción de las transferencias e innovaciones tecnológicas; a formar profesionales responsables, con conciencia ética y solidaria, capaces de contribuir al desarrollo del país, a la vigencia del orden democrático y a estimular la participación social.

El Estado, a través de la Secretaría Nacional de Educación Superior, Ciencia y Tecnología (SENESCYT) y de la LOES, propicia el mejoramiento de la calidad de este sistema, consciente de que las universidades ecuatorianas conforman un motor fundamental en la transformación de la sociedad.

El artículo 351 de la Constitución de la República del Ecuador sostiene que "El Sistema de Educación Superior estará articulado al Sistema Nacional de Educación y al Plan Nacional de Desarrollo; la ley establecerá los mecanismos de coordinación del Sistema de Educación Superior con la Función Ejecutiva. Este sistema se regirá por los principios de autonomía responsable, cogobierno, igualdad de oportunidades, calidad, pertinencia, integralidad, autodeterminación para la producción del pensamiento y conocimiento, en el marco del diálogo de saberes, pensamiento universal y producción científica tecnológica global”.(16)

Es de destacarse que las universidades, al igual que el resto de las instituciones y sectores en los distintos niveles de gobierno, han de articular la formulación de sus políticas, planificación, programas, proyectos públicos y la inversión y asignación de recursos, con los objetivos, políticas, lineamientos estratégicos, metas y estrategias territoriales establecidas en el Plan Nacional para el Buen Vivir 2013 - 2017, así como con los lineamientos y directrices de la Estrategia Territorial Nacional y de las Agendas Zonales respectivas. Estos importantes documentos rectores, derivados de la nueva Constitución, constituyen la plataforma orientada por el Estado, que expresa la voluntad de continuar con la transformación histórica del Ecuador. Los objetivos del Plan Nacional del Buen Vivir son: “...consolidar el Estado democrático y la construcción del poder popular; auspiciar la igualdad, la cohesión, la inclusión y la equidad social y territorial, en la diversidad; mejorar la calidad de vida de la población; fortalecer las capacidades y potencialidades de la ciudadanía; construir espacios de encuentro común y fortalecer la identidad nacional, las identidades diversas, la plurinacionalidad y la interculturalidad; consolidar la transformación de la justicia y fortalecer la seguridad integral, en estricto respeto a los derechos humanos; garantizar los derechos de la naturaleza y promover la sostenibilidad territorial y global; consolidar el sistema económico social y solidario, de forma sostenible; garantizar el trabajo digno en todas sus formas; impulsar la transformación de la matriz productiva; asegurar la soberanía y eficiencia de los sectores estratégicos para la transformación industrial y tecnológica; garantizar la soberanía y la paz; y, profundizar la inserción estratégica en el mundo y la integración latinoamericana".(17)

La LOES incluye la creación del Consejo de Educación Superior (CES). En su artículo 169, se establecen las atribuciones y deberes de este organismo, entre las que prescribe la aprobación de los reglamentos de régimen académico y títulos; de régimen de postgrado; $y$ de las diferentes modalidades de estudios, entre otros. De igual forma, la Ley incluye la creación del Centro de Evaluación, Acreditación y Aseguramiento de la Calidad de la Educación Superior (CEAACES), como organismo responsable de normar la autoevaluación institucional y ejecutar los procesos de evaluación externa, acreditación y el aseguramiento de la calidad.

Ambos órganos constituyen un importante impulso en la sensibilización de la necesidad de priorizar el perfeccionamiento del desempeño profesional de los docentes universitarios, por cuanto, aseguran la calidad de la educación superior y el fomento de procesos permanentes de mejoramiento de la calidad académica y de gestión en 
los Centros de este nivel. Desde ellos, se instrumentan los criterios pedagógicos y operativos de la LOES para jerarquizar la calidad de la educación universitaria y por la pertinencia, integralidad y autodeterminación de sus procesos.

Por primera vez en el Ecuador, desde lo legal, se unificaron criterios orientados a la calidad de los procesos universitarios, analizados, integralmente, en función de la formación de profesionales que respondan por los valores democráticos y la participación social que caracterizan a la sociedad ecuatoriana que se construye. De igual forma, en función de la calidad de la educación superior, por primera vez, se jerarquizó, jurídicamente, la precisión de los derechos de los profesores universitarios, entre los que se incluyó el de recibir formas de superación que los capaciten, tanto en sus áreas de formación como en el área pedagógica.

La caracterización lograda desde lo histórico - lógico posibilita valorar que, a diferencia de etapas anteriores, en las que no se le ofreció la suficiente atención a la calidad del desempeño profesional pedagógico de los profesores universitarios, las transformaciones que se han estado dando en los últimos siete años en la sociedad ecuatoriana, han determinado una evolución en la manera de pensar y de concebir dicho desempeño y su mejoramiento. Sin embargo, se comienza a valorar nuevas exigencias en el desempeño profesional pedagógico de los profesores universitarios que revelan un cambio hacia la calidad y la excelencia en la educación superior.

En el Ecuador, los requerimientos crecientes del mercado; los cambios políticos, sociales y económicos imperantes; el impetuoso llamado al incremento del protagonismo de las universidades en la transformación de su entorno y de su compromiso con el desarrollo local y de toda la sociedad, en general; la intensa preocupación por la evaluación institucional y la sensibilización de sus propios docentes y directivos por optimizar los recursos y mejorar la calidad del servicio que prestan, apuntan a una nueva universidad orientada hacia la máxima calidad.

Bajo estos presupuestos, la elevación de la calidad de la educación superior está llamada a convertirse en una realidad concreta en el Ecuador. De esta manera, tal como propone Méndez E, “... se lograría desarrollar una universidad socialmente efectiva, que asegure, por intermedio de sus profesionales y especialistas, la producción científico-cultural y la acción directa de sus miembros, la atención a las necesidades y aspiraciones de las comunidades y desarrollar una universidad en la que se inviertan, racionalmente, y oportuna- mente, los recursos humanos y materiales necesarios para alcanzar los objetivos de la institución. Ello es, lograr una universidad de calidad integral y de calidad en cada uno de sus procesos y productos académicos".(18)

Diferentes estudios refieren la necesidad de que el cambio en la educación superior esté acompañado de procesos de renovación y/o actualización de sus profesores. "El objetivo principal de la educación superior es mejorar la calidad de todos sus procesos y entre los indicadores más importantes a través de los cuales ésta se mide, está: la calidad de los egresados y del claustro. Aquí, se destaca la importancia social del profesor universitario en el curso de los procesos en marcha".(19)

Organismos preocupados por la formación y la superación de los docentes y comprometidos con la calidad de los procesos formativos en las universidades, han generado reuniones de los ministros de Educación Superior de América Latina y el Caribe. En la segunda reunión intergubernamental del Proyecto Regional de Educación Superior para América Latina y el Caribe, convocada por la UNESCO y realizada en Buenos Aires, se insistió en la necesidad de intensificar los esfuerzos para obtener una educación de calidad y se reconoció la prioridad que deben tener la formación y la superación pedagógica de los docentes entre las direcciones de trabajo para lograrlo. En dicha reunión, se recomendó: “....instalar el tema docente como un aspecto prioritario en la agenda de las políticas públicas; institucionalizar espacios y mecanismos que propicien la participación de los docentes en el diseño y desarrollo de las políticas educativas y del currículo y en la gestión de los centros educativos superiores y promover una educación inicial y continua que responda a las exigencias de la sociedad del siglo XXI, y que desarrolle en los docentes las competencias necesarias para trabajar".(20)

Además, se propuso “...fortalecer carreras docentes orientadas al desarrollo profesional y al mejoramiento del desempeño para atraer y retener a los mejores profesores, así como desarrollar y mejorar sistemas objetivos de evaluación del desempeño docente orientados a su desarrollo profesional".(21)

Como se aprecia, en el último decenio se han privilegiado los esfuerzos encaminados al mejoramiento de la calidad de la educación superior, y en ese empeño se ha identificado la variable desempeño profesional del profesor como un factor decisivo para lograr el salto cualitativo que requiere.

Hoy, se aprecia un cierto consenso en la idea de 
que el fracaso o el éxito de todo sistema educativo depende, fundamentalmente, de la calidad del desempeño de sus docentes. Es evidente, la necesidad de profundizar en los procesos de formación y superación sobre la base del diagnóstico de las necesidades individuales y colectivas de los profesores. En este sentido, resulta necesario establecer alternativas en las universidades que aseguren un saber más integrado en el orden pedagógico y en el de su especialidad, y que los docentes universitarios asuman su superación a lo largo de toda su carrera profesional.

Lo antes planteado, se corresponde con los principios reconocidos en la LOES, como criterios que rigen de manera integral a las instituciones, los actores, los procesos, las normas, los recursos, y demás componentes del sistema. En el artículo 156 de esta Ley se enuncia el aseguramiento del perfeccionamiento del desempeño de los profesores y que "...los presupuestos de las instituciones del Sistema de Educación Superior constarán de partidas destinadas a financiar planes de becas y ayudas económicas para la especialización y año sabático".(22)

Estas proyecciones legales apoyan el reconocimiento del docente en los procesos emprendidos en el Ecuador para elevar la calidad de la educación superior. En general, como tendencia, el mejoramiento del desempeño profesional pedagógico se analiza, actualmente, desde enfoques más integrales y sistémicos. Se da respuesta así, a una deuda histórica con los profesores universitarios y sus necesidades de superación y autoperfeccionamiento.

A través del desarrollo de la educación permanente y otras vías de superación al alcance de las universidades ecuatorianas, éstas han de lograr desempeños en los docentes que aseguren la máxima calidad, en correspondencia con las exigencias de la LOES y los estándares e indicadores de calidad planteados como parte de los procesos de evaluación y acreditación.

Los estándares de calidad implican nuevas exigencias a todos los profesores, al constituir las “...descripciones de logros esperados de los diferentes actores e instituciones del sistema educativo, (...) orientaciones de carácter público que señalan las metas educativas para conseguir una educación de calidad".(23) En el caso concreto de los estándares de calidad del desempeño docente, planteados por el Ministerio de Educación, “... son descripciones de lo que debe hacer un profesor competente; es decir, de las prácticas pedagógicas que tienen más correlación positiva con el aprendizaje de los estudiantes".(24)
En general, estos estándares se corresponden con los indicadores de evaluación que contempla el Modelo de Evaluación de Carreras Presenciales y Semipresenciales de las Universidades y Escuelas Politécnicas del Ecuador (25) que, actualmente, aplica el CEAACES.

En este documento, específicamente en el criterio Academia, se reconoce que, los profesores universitarios "...deben tener las competencias necesarias para cubrir todas las áreas curriculares de las carreras, (...) para mantener niveles adecuados de interacción estudiantes-profesores, (...) desempeñar actividades tutoriales, actividades de servicio a la comunidad, interactuar con los sectores productivos o de servicio y asegurar las prácticas de los estudiantes".(26)

El Modelo, también, incluye indicadores referidos a la demostración de suficiencia para desarrollar e implementar procesos de evaluación y mejoramiento continuo de las Carreras, la consecución de sus objetivos, así como los resultados de aprendizaje. De igual forma, en el criterio Calidad de los profesores, incluyen indicadores referidos a la experiencia en el ejercicio profesional, en la investigación, así como la formación pedagógica del cuerpo docente de las Carreras. "Las competencias generales de los profesores se pueden apreciar, mediante, factores tales como: su nivel de escolaridad, formación académica especializada de posgrado, actualización científica, producción de artículos o trabajos científicos publicados en revistas que figuran en bases de datos, su experiencia profesional, su experiencia y efectividad en la enseñanza, su habilidad para comunicarse, su entusiasmo para desarrollar programas más efectivos, el empleo de las tecnologías y su participación en redes y sociedades profesionales."(27)

Estos indicadores, integran los conocimientos, habilidades y valores propios del desempeño profesional pedagógico que se espera de los profesores universitarios ecuatorianos, en la actualidad, y que también, han sido considerados como premisas para la introducción del nuevo Reglamento de Régimen Académico (28), establecido por el CES, y que han podido ser ampliadas y profundizadas en los diferentes talleres realizados en el proceso de acompañamiento de ese Órgano para su implementación en las universidades.

Lo analizado hasta aquí, evidencia que la proyección hacia la calidad de los procesos e instituciones del Sistema de Educación Superior implican nuevas exigencias a todas las universidades y a sus claustros. En correspondencia, estas han de 
contar con estrategias que faciliten el mejor desempeño profesional pedagógico de los profesores y que puedan ejercer el papel que les corresponde en la transformación socioeducativa planteada en la Constitución del Ecuador.

Esta proyección supone un análisis de la formación de los profesores universitarios, incluyendo su formación pedagógica, así como de sus condiciones internas de compromiso y autonomía. Sin embargo, en Ecuador, la formación exigida para ingresar como profesor universitario solo requiere de conocimientos en el área del saber, en la cual se ha de desempeñar, expresándose una contradicción entre los retos que implican la LOES y el resto de los documentos normativos referidos, y la situación real que caracteriza la preparación pedagógica de los profesores.

Esta preparación resulta insuficiente, lo que es considerado por el CES como uno de los nodos críticos o problemas de carácter nacional que deben generar la intervención para el mejoramiento de la calidad de la Educación Superior del Ecuador. Entre los criterios que, en este sentido, plantea el CES, están:

- $\quad$ "Los docentes de las universidades, en su mayoría, no cumplen con los niveles de cualificación y titulación que la LOES exige.

- $\quad$ "Hay escasas iniciativas para el apoyo al docente en sus actividades de creación de ambientes de aprendizajes, narrativas pedagógicas, académicas y científicas.

- $\quad$ No existen procesos de habilitación de los docentes para su buen desempeño en las distintas fases curriculares y niveles de aprendizaje.

- "Reducidas iniciativas de formación de cuerpos o colectivos académicos con procesos de investigación y gestión del conocimiento y los aprendizajes".(29)

\section{Conclusiones}

El estudio de la evolución histórica de la evaluación en la educación superior y del desempeño profesional pedagógico y su mejoramiento en el Ecuador, revela que, como tendencia, en las diferentes etapas del desarrollo del país, no se le ofreció la suficiente atención a este aspecto, hasta el año 2007, según Decreto No.3782 de 2 de Octubre del 2007, en que se orientó una verdadera política oficial para su desarrollo y control. Con el actual gobierno, por primera vez, desde lo legal, se unificaron criterios orientados a la elevación de la calidad de la educación superior de acuerdo con la sociedad que se construye.

\section{Referencias Bibliográficas}

1. Los Jesuitas y su extrañamiento del Reino de Quito. En: Los Jesuitas en el Ecuador III [Seriada en línea]; 2007. p. 27. Disponible en: http://www2.jesuitas.ec/. (Consultado diciembre 10, 2012).

2. Los Jesuitas y su extrañamiento del Reino de Quito. En: Los Jesuitas en el Ecuador III [Seriada en línea]; 2007. p. 32. Disponible en: http://www2.jesuitas.ec/ (Consultado diciembre 10, 2012).

3. Período de Consolidación (1605 - 1767). En: Los Jesuitas en el Ecuador II [Seriada en línea]; 2007. p. 20. Disponible en: http://www2.jesuitas.ec/. (Consultado diciembre 10, 2012).

$4 . \quad$ Ley Orgánica de Instrucción Pública. [Versión digital en pdf]. Quito, Ecuador: Imprenta de la "Gutemberg"; 1906. Disponible en: http://www. oei.es/quipu/ecuador/ecu02.pdf/. (Consultado noviembre 17, 2012).

5. Ayala E. Lucha política y origen de los partidos en el Ecuador. Quito, Ecuador: CEN-TEHIS; 1988.

6. Ayala E. Lucha política y origen de los partidos en el Ecuador. Quito, Ecuador: CEN-TEHIS; 1988. p. 129.

7. Pareja F. La Educación Superior en el Ecuador. Caracas, Venezuela: Centro Ecuatoriano de Investigaciones Sociales (CEIES); 1986. CRESALC- UNESCO. Disponible en: http:// www.unesdoc.unesco.org/images/0007/000726/ 072628so.pdf/. (Consultado noviembre 17, 2012). 8. Abendaño A. Procesos de formación de los docentes por parte de universidades e institutos pedagógicos en Ecuador. Quito, Ecuador: Digital Observatory for Higher Education in Latin America and Caribbean IESALC; 2004. p. 7. Reports available at www.iesalc.unesco.org.ve/. (Consultado noviembre 7, 2012).

9. Abendaño A. Procesos de formación de los docentes por parte de universidades e institutos pedagógicos en Ecuador. Quito, Ecuador: Digital Observatory for Higher Education in Latin America and Caribbean IESALC; 2004. Reports available at www.iesalc.unesco.org.ve/. (Consultado noviembre 17, 2012).

10. Ley Orgánica de Educación Superior. Quito, Ecuador: Ley No. 16. RO/ 77 de 15 de Mayo del 2000. Congreso Nacional; 2000, Disponible en: http://www.oei.es/quipu/ecuador.pdf/. (Consultado septiembre 24, 2012).

11. Ley Orgánica de Educación Superior. Quito, Ecuador: Ley No. 16. RO/ 77 de 15 de Mayo del 2000. Congreso Nacional; 2000. Art. 1, literal b. p. 1. Disponible en: http://www.oei.es/quipu/ ecuador.pdf/ (Consultado septiembre 24, 2012).

12. Macías W. El Sistema de Educación Superior del Ecuador. Red de Observatorios de Bue- 
nas Prácticas de Dirección Estratégica Universitaria en América Latina y Europa. [Versión digital en pdf]. Guayaquil, Ecuador: Escuela Superior Politécnica del Litoral; 2011. p. 24.

13. Abendaño A. Procesos de formación de los docentes por parte de universidades e institutos pedagógicos en Ecuador. Quito, Ecuador: Digital Observatory for Higher Education in Latin America and Caribbean IESALC; 2004. p. 22. Reports available at www.iesalc.unesco.org.ve. (Consultado noviembre 17, 2012).

14. Constitución de la República del Ecuador. Asamblea Nacional. Publicada en el Registro Oficial No. 449; 20 de octubre de 2008. p. 23.

15. Ley Orgánica de Educación Superior. Quito, Ecuador: Presidencia de la República, Registro Oficial No 298; 2010. p. 5. Disponible en: http://www. documentacion.asambleananacional. gov.ec./ (Consultado septiembre 23, 2012).

16. Constitución de la República del Ecuador. Asamblea Nacional. Publicada en el Registro Oficial No. 449; 20 de octubre de 2008. p. 127.

17. Plan Nacional de Desarrollo / Plan Nacional para el Buen Vivir 2013-2017: Secretaría Nacional de Planificación y Desarrollo (Senplades). [Versión digital en pdf]. Quito, Ecuador; 2013. pp. 15-16.

18. Méndez E. Gerencia Académica. La construcción de la educación del III Milenio. Maracaibo, Venezuela: Universidad del Zulia; 1993. p. 47.

19. Soriano M. El profesor universitario ante los retos del mundo de hoy: sus competencias laborales. Instituto Superior Politécnico José Antonio Echeverría. Contexto Educativo. Número 30 Año VI. [Versión digital en pdf]. La Habana, Cuba; 2000. p. 4.

20. UNESCO. Educación y nuevas tecnologías. Uso de las TIC como herramientas de una educación inclusiva y de calidad; 2007. p.132. Disponible en: www.workman.cl/unesco/informe_ seminario_escuelas_asociadas_2007 [Versión digital en pdf]. (Consultado diciembre 26, 2012).

21. UNESCO. Educación y nuevas tecnologías. Uso de las TIC como herramientas de una educación inclusiva y de calidad; 2007. p.6. Disponible en: www.workman.cl/unesco/informe_seminario_escuelas_asociadas_2007 [Versión digital en pdf]. (Consultado diciembre 26, 2012).

22. Ley Orgánica de Educación Superior. Quito, Ecuador: Presidencia de la República, Registro Oficial No 298; 2010. p. 24. Disponible en: http://www. documentacion.asambleananacional. gov.ec./ (Consultado septiembre 23, 2012).

23. Estándares de desempeño profesional docente. Propuesta para la discusión ciudadana. Quito, Ecuador: Ministerio de Educación. Documento de Propuesta [Seriada en línea]; febrero de 2011. p. 2. Disponible en: http://www. psicologia- porlavida.blogsport.com.pdf/. (Consultado junio 9, 2012).

24. Estándares de desempeño profesional docente. Propuesta para la discusión ciudadana. Quito, Ecuador: Ministerio de Educación. Documento de Propuesta [Seriada en línea]; febrero de 2011. p. 7. Disponible en: http://www. psicologiaporlavida.blogsport.com.pdf/. (Consultado junio 9, 2012).

25. Modelo de Evaluación de Carreras Presenciales y Semipresenciales de las Universidades y Escuelas Politécnicas del Ecuador. Quito, Ecuador: Consejo de Evaluación, Acreditación y Aseguramiento de la Calidad de la Educación Superior. CEAACES; agosto de 2013. Disponible en: http://www. ceaaces.gob.ec/. (Consultado octubre 7, 2013).

26. Modelo de Evaluación de Carreras Presenciales y Semipresenciales de las Universidades y Escuelas Politécnicas del Ecuador. Quito, Ecuador: Consejo de Evaluación, Acreditación y Aseguramiento de la Calidad de la Educación Superior. CEAACES; agosto de 2013. pp. 7-8. Disponible en: http://www.ceaaces.gob.ec/. (Consultado octubre 7, 2013).

27. Modelo de Evaluación de Carreras Presenciales y Semipresenciales de las Universidades y Escuelas Politécnicas del Ecuador. Quito, Ecuador: Consejo de Evaluación, Acreditación y Aseguramiento de la Calidad de la Educación Superior. CEAACES; agosto de 2013. pp. 8-9. Disponible en: http://www.ceaaces.gob.ec/. (Consultado octubre 7, 2013).

28. Reglamento de Régimen Académico. Quito, Ecuador: Consejo de Educación Superior. CES; noviembre de 2013. Disponible en: http:// www.ces.gob.ec/. (Consultado noviembre 17, 2013).

29. Larrea E. El currículo de la Educación Superior desde la complejidad sistémica. Algunas consideraciones para orientar el proceso de construcción del nuevo modelo de formación universitaria. Guayaquil, Ecuador: Primer Taller del Plan de acompañamiento del CES para la implementación del Reglamento de Régimen Académico. CES; 2014. p.13. Disponible en: http://www.ces. gob.ec/. (Consultado febrero 3, 2014).

\section{Bibliografía}

1. Guerra D. Educación y cambio estructural. Colección Reflexión u Análisis. México: Editores Noriega; 2000.

2. Sánchez J A. El desarrollo profesional del docente universitario. Madrid, España: Universidad Politécnica de Madrid [Seriada en línea]; s/f. Disponible en: http:/www.lachacana.ec/ (Consultado diciembre 9, 2012).

3. Ortega M. Situación actual y perspectiva 
del desempeño docente en la Escuela de Sanidad de la Armada. Programa de Maestría en Gerencia Educativa. Quito, Ecuador: Universidad Andina Simón Bolívar, Sede Ecuador; 2009.

$4 . \quad$ Salazar H. Formación humana del docente latinoamericano. Educación Superior. Revista Pedagógica Semestral. Año 5. No. 9, Esmeraldas, Ecuador; 2008.

$5 . \quad$ La formación del docente universitario. Informe. Madrid, España: Ministerio de Educación y Ciencia (MEC); 1992. p. 35.

6. Sánchez J. El desarrollo profesional del docente universitario. Madrid, España: Universidad Politécnica de Madrid [Seriada en línea]; s/f. p.14. Disponible en: http:/www.lachacana.ec/. (Consultado diciembre 12, 2012).

7. Añorga J, Valcárcel N. La Profesionalización y Educación Avanzada. Sucre, Bolivia: Material Docente. Maestría y Doctorado CENESEDA-ISPJEV [En soporte electrónico]; 1995. p. 10. Disponible en: http:/www.cujae.educ.cu/. (Consultado septiembre 27, 2011).

8. Campoverde E. Algunos antecedentes históricos del desarrollo del desempeño profesional pedagógico del profesor universitario y su mejoramiento en el contexto del Ecuador. Revista IPLAC. No.1, ene - febr de 2013.

9. Colectivo de Autores Cubanos del ICCP. Caracterización del desempeño profesional del docente de preuniversitario. [Informe de resultado, Proyecto: Un modelo para el desempeño profesional del docente de preuniversitario]. La Habana, Cuba: Instituto Central de Ciencias Pedagógicas; 2007.

10. Huerta J. Desarrollo curricular por competencias profesionales integrales. 2007. Disponible en http://www.comsoc.udg.mx/. (Consultada noviembre 17, 2013).

11. Lamas M. La superación de los profesores de Historia como promotores del desarrollo de la formación humanista en la Escuela Politécnica. [Tesis en opción al Grado Científico de Doctor en Ciencias Pedagógicas]. La Habana, Cuba: Instituto Superior Pedagógico Enrique José Varona; 2006.

12. Lazo J. Universidad-Sociedad. Curso Internacional de Maestría en Educación Superior. Sucre, Bolivia: Universidad Andina Simón Bolívar; 1994.

13. Marzano R J. The art and science of teaching: A comprehensive framework for effective instruction: Alexandria, VA: ASCD; 2007. 